\title{
ON FINITELY GENERATED SUBGROUPS OF A FREE GROUP
}

A. KARRASS AND D. SOLITAR

1. Introduction. In [2], M. Hall, Jr. proved the following theorem: Let $H$ be a finitely generated subgroup of a free group $F$ and suppose $\beta_{1}, \cdots, \beta_{n}$ are in $F$ but no $\beta_{i}$ is in $H$. Then we may construct a subgroup $\bar{H}$ of finite index in $F$ containing $H$ and not containing any $\beta_{i}$.

Now the proof in [2] actually shows more, viz., that $H$ is a free factor of $\bar{H}$. In particular, taking the set of $\beta_{i}$ 's to be empty one obtains the following:

If $H$ is a finitely generated subgroup of a free group $F$, then $H$ is a free factor of a subgroup $\bar{H}$ of finite index in $F$.

In this paper we shall show how a number of results about finitely generated subgroups of a free group follow in a natural way from the above special case of the theorem of M. Hall, Jr. In particular, we derive the following: a finitely generated subgroup $H$ is of finite index in $F$ iff $H$ has a nontrivial intersection with every nontrivial normal subgroup of $F$ (this includes the case, see [4], where $H$ contains a nontrivial normal subgroup of $F$, and the case, see L. Greenberg [1], when $H$ contains a nontrivial subnormal subgroup of $F$ ); a generalization of this for a pair of subgroups $H, K$ (see Theorem 3, Corollary 1); other types of conditions for a finitely generated $H$ to be of finite index in $F$ (first proved by L. Greenberg in [1] for discrete groups of motions of the hyperbolic plane (which include free groups); and Howson's result that the intersection of two finitely generated subgroups of $F$ is finitely generated. We also derive a quick way of obtaining the precise index of $H$ in $F$ from inspection of a Nielsen reduced set of generators for $H$.

2. The results. In what follows "f.g." denotes "finitely generated" and "f.i." denotes "finite index."

If $H, \bar{H}$ are subgroups of a group $G$, then $H$ is a factor of $\bar{H}$ if $\bar{H}$ is the free product $H * H_{1}$ for some $H_{1}<G ; \bar{H}$ is a completion of $H$ in $G$ if $H$ is a factor of $\bar{H}$ and $\bar{H}$ is of f.i. in $G$. By the above result of M. Hall, Jr., every f.g. subgroup $H$ of a free group $F$ has a completion $\bar{H}$ in $F$.

Lemma 1. Let $H$ be a factor of $\bar{H}$ in $G$ and let $K<G$. Then $H \cap K$ is a factor of $\bar{H} \cap K$.

Received by the editors October 18, 1968. 
Proof. Since $\bar{H}$ is a free product, say $\bar{H}=H * H_{1}$, we may apply the Kurosh subgroup theorem to the subgroup $\bar{H} \cap K$ and conclude that $\bar{H} \cap K$ is a free product of factors one of which is its intersection with $H$, viz., $H \cap(\bar{H} \cap K)=H \cap K$. [Remark: If $G$ is a free group, then Lemma 1 can be proved without using the Kurosh subgroup theorem; see, for example, [6, p. 117 exercise 32].]

COROLlaRy. If $\bar{H}, \bar{K}$ are completions of $H, K$ respectively in $G$, then $\bar{H} \cap \bar{K}$ is a completion of $H \cap K$ in $G$.

Proof. Clearly $\bar{H} \cap \bar{K}$ is of f.i. in $G$. Moreover, by Lemma $1, H \cap K$ is a factor of $\bar{H} \cap K$ which itself is a factor of $\bar{H} \cap \bar{K}$.

TheOREM 1 (Howson). If $H, K$ are f.g. subgroups of a free group, then $H \cap K$ is also f.g.

Proof. Let $\bar{H}$ be a completion of $H$ in $F$. Then $\bar{H} \cap K$ is of f.i. in $K$ and so is f.g. Moreover, since $H \cap K$ is a factor of $\bar{H} \cap K$ (by Lemma 1), we have that $H \cap K$ is a homomorphic image of $\bar{H} \cap K$ and so $H \cap K$ is f.g.

Lemma 2. If $G$ is the free product $A * B(B \neq 1)$ and $N$ is a subnormal subgroup of $G$ contained in $A$, then $N=1$.

Proof. Suppose $L \neq 1$ is a subgroup of $A$. Then the normalizer of $L$ in $G$ is contained in $A$, for $v^{-1} a v$ is in $A$ implies $v$ is in $A$ (as is easily seen by using the reduced form of $v$ in the free product). Now suppose

$$
N_{r} \triangleleft N_{r-1} \triangleleft \cdots \triangleleft N_{1} \triangleleft G
$$

and $N_{r} \neq 1, N_{r}<A$. Then $N_{i-1}$ is contained in the normalizer of $N_{i}$ and therefore $G<A$, contrary to $B \neq 1$.

TheOREM 2. (GREENBERG). If $H$ is a f.g. subgroup of a free group $F$ and $H$ contains a subnormal subgroup $N \neq 1$ of $F$, then $H$ is of $f . i$. in $F$.

Proof. Let $\bar{H}$ be a completion of $H$ in $F$. Then $N<\bar{H}$ and $N$ is a subnormal subgroup of $\bar{H}=H * H_{1}$. Hence by Lemma 2, $H_{1}=1$ and so $H=\bar{H}$, i.e. $H$ is of f.i. in $F$.

Lemma 3. If $G=A * B(A \neq 1, B \neq 1)$, then $A$ has trivial intersection with some noncyclic normal subgroup of $G$, viz., the normal subgroup of $G$ generated by $B$.

Proof. Let $a \in A, b \in B$ with $a \neq 1, b \neq 1$, and let $N$ be the normal subgroup of $G$ generated by $B$. Then under the natural homomorphism of $G \rightarrow G / N, A$ is mapped isomorphically onto itself and so $A \cap N=1$. Moreover, since $b$ and $a b a^{-1}$ are in $N$ and do not commute, $N$ is noncyclic. 
Theorem 3. Let $H$ be a f.g. subgroup $\neq 1$ of a free group $F$. Then the following conditions are equivalent:

(a) $H$ is of f.i. in $F$.

(b) $H$ has a nontrivial intersection with every nontrivial normal subgroup of $F$.

(c) $H$ is not properly contained in a subgroup of rank larger than that of $H$.

Proof. Clearly (a) implies that $H$ has nontrivial intersection with every infinite subgroup and so (a) implies (b); (a) implies (c) follows from the Schreier rank formula, since $j(n-1)+1 \geqq n$ (see, for example, [6, p. 104]).

Conversely, suppose (c). Hence if $\bar{H}=H * H_{1}$ is a completion of $H$ in $F$, then $H_{1}=1$, and $H=\bar{H}$ is of f.i. in $F$.

Finally, suppose (b). Again let $\bar{H}=H * H_{1}$ be a completion of $H$ in $F$ and suppose $H_{1} \neq 1$. Let $N$ be the normal subgroup of $\bar{H}$ generated by $H_{1}$. By Lemma $3, N \cap H=1$. Since the normalizer of $N$ in $F$ contains $\bar{H}, N$ has finitely many conjugates in $F$. The intersection of these conjugates is therefore nontrivial (see, for example, [5, p. 219]), is normal in $F$, and is disjoint from $H$, contrary to hypothesis. Consequently, $H_{1}=1$ and $H=\bar{H}$ is of f.i. in $F$.

[Theorem 3 is a generalization of Theorem 2 because any two nontrivial subnormal subgroups of a free product intersect nontrivially. For, let $N, K$ be nontrivial normal subgroups of $G$. Suppose $N \cap K=1$; then every element of $N$ commutes with every element of $K$. If $N$ contains an element $\neq 1$ in $A$, then $K<A$ (see, for example, [6, Corollary 4.1.6]), contrary to Lemma 2 . Hence we may assume $N$ and $K$ have trivial intersection with the conjugates of $A$ and $B$. Then the elements of $N$ and $K$ are of infinite order and $\exists n \in N, k \in K, n \neq 1$, $k \neq 1$, such that $n k=k n$ and so $n, k$ are both powers of the same element. But then $N \cap K \neq 1$.

Next suppose

$$
1 \neq N_{r} \triangleleft N_{r-1} \triangleleft \cdots \triangleleft N_{1} \triangleleft G
$$

and

$$
1=K_{s} \triangleleft K_{8-1} \triangleleft \cdots \triangleleft K_{1} \triangleleft G .
$$

We show by induction on $r+s$, the total length of the two normal chains, that $N_{r} \cap K_{s} \neq 1$. If $r+s=2$, then $N_{r}$ and $K_{s}$ are normal in $G$. Suppose $r+s>2$, and say $s \geqq 2$. Since $N_{r}$ and $K_{1}$ occur in normal chains of total length $r+1<r+s$, by inductive hypothesis $N_{r} \cap K_{1} \neq 1$. Moreover, $K_{1}$ is either infinite cyclic or a proper free product (by Lemma 2 and the Kurosh subgroup theorem). Hence, since in $K_{1}$, 
$N_{r} \cap K_{1}$ and $K_{s}$ occur in normal chains of total length $r+s-1$, we have $\left(N_{r} \cap K_{1}\right) \cap K_{s}=N_{r} \cap K_{s} \neq 1$.]

Corollary 1. Let $H, K$ be subgroups of a free group $F$ with $H$ f.g. Then the following conditions are equivalent:

(a) $H \cap K$ is of f.i. in $K$.

(b) $H$ contains a nontrivial subnormal subgroup $N$ of $K$.

(c) $H$ has a nontrivial intersection with every nontrivial normal subgroup of $K$.

Proof. Clearly (a) implies (b) and (c).

Suppose (b). Let $\bar{H}$ be a completion of $H$ in $F$. Then $H \cap K$ contains $N$ and $N$ is subnormal in $\bar{H} \cap K$. By Lemma $1, H \cap K$ is a factor of $\bar{H} \cap K$ and so by Lemma $2, \bar{H} \cap K=H \cap K$. Clearly $\bar{H} \cap K$ is of f.i. in $K$.

Suppose (c) and again let $\bar{H}$ be a completion of $H$ in $F$. Then $\bar{H} \cap K$ $=(H \cap K) * L_{1}$ and arguing as in the proof of the above theorem we obtain that $H \cap K=\bar{H} \cap K$, which is of f.i. in $K$.

Corollary 2 (Greenberg). Let $H \neq 1$ be a f.g. subgroup of a free group $F$. Then the following conditions are equivalent:

(a) $H$ is of f.i. in $F$.

(b) $H$ has a nontrivial intersection with every noncyclic subgroup of $F$.

(c) $H$ is not contained in a subgroup of infinite rank.

Proof. Clearly (a) implies (b) and (c).

Conversely, suppose (b). Since every nontrivial normal subgroup of a noncyclic free group is noncyclic, $H$ satisfies (b) of the above theorem and hence (a) holds.

Finally, suppose (c). Let $\bar{H}=H * H_{1}$ be a completion of $H$ in $F$. If $H_{1} \neq 1$ then $N$, the normal subgroup of $\bar{H}$ generated by $H$, has infinite index in $\bar{H}$ and therefore is infinitely generated.

The terminology in the following theorem is used in the same way as in $\$ 3.2$ of [6].

TheOREM 4. Suppose $F$ is the free group on $x_{1}, x_{2}, \cdots, x_{n}$ and $H$ is a f.g. subgroup of $F$ with a Nielsen reduced set of generators $\left\{W_{i}\left(x_{v}\right)\right\}$, $1 \leqq i \leqq r$, where the right halves of the $W_{i}$ of even length are isolated. Let

$$
W_{i}=U_{i} x_{\nu_{i}}^{e_{i}} V_{i}^{-1}
$$

where $V_{i}^{-1}$ is the minor terminal segment of $W_{i}$, and let $j$ be the number of distinct initial segments of $U_{1}, V_{1}, \cdots, U_{r}, V_{r}$. Then $H$ is of f.i. in $F$ iff $j=(r-1) /(n-1)$ iff $[F: H]=j$. 
Proof. The argument given by M. Hall, Jr. in [2] to prove the result quoted in the introduction can be modified to construct a Schreier coset representative function whose representatives are precisely the distinct initial segments of $U_{1}, V_{1}, \cdots, U_{r}, V_{r}$ and such that the representative of $U_{i} x_{\nu_{i}}^{\epsilon_{i}}$ is $V_{i}$. The associated subgroup $\bar{H}$ clearly has index $j$ in $F$ and has $H$ as a factor. Now $H$ is of f.i. in $F$ iff $H=\bar{H}$ iff the rank of $H$ equals the rank of $\bar{H}$. But the rank of $\bar{H}$ is $j(n-1)+1$. Therefore $H$ is of f.i. in $F$ iff $r=j(n-1)+1$, i.e., $j=(r-1) /(n-1)$.

Note that a given Nielsen reduced set of generators $\left\{W_{i}\left(x_{\nu}\right)\right\}$ may be converted into one with isolated right halves simply by replacing $W_{j}$ by $W_{j}^{-1}$ if necessary. The above test requires that the right halves be isolated as the following example shows: Let $F$ be the free group on $a, b$ and let $H=g p\left(a^{2}, a b, a b^{-1}\right)=g p\left(a^{2}, a b, b a^{-1}\right)$. Then both sets of generators for $H$ are Nielsen reduced. Now in this case $n=2, r=3$ and so by the above test $H$ is of f.i. iff $j=2$. The first set of generators has isolated right halves and $U_{1}=a, V_{1}=1, U_{2}=a, V_{2}=1, U_{3}=a$, $V_{3}=1$, so that $j=2$; hence $H$ is of f.i. 2 (indeed, $H$ is just the subgroup consisting of words of even length in $F$ ). On the other hand, the second set of generators does not have isolated right halves and an attempt to apply the test in this case yields $U_{1}=a, V_{1}=1, U_{2}=a$, $V_{2}=1, U_{3}=b, V_{3}=1$, which leads to $j=3$ and the incorrect conclusion that $H$ is not of f.i.

\section{REFERENCES}

1. L. Greenberg, Discrete groups of motions, Canad. J. Math., 12 (1960), 414-425.

2. M. Hall, Jr., Coset representations in free groups, Trans. Amer. Math. Soc. 67 (1949), 421-432.

3. A. G. Howson, On the intersection of finitely generated free groups, J. London Math. Soc. 29 (1954), 428-434.

4. A. Karrass and D. Solitar, Note on a theorem of Schreier, Proc. Amer. Math. Soc. 8 (1957), 696-697.

5. - On free products, Proc. Amer. Math. Soc. 9 (1958), 217-221.

6. W. Magnus, A. Karrass and D. Solitar, Combinatorial group theory, Interscience, New York, 1966.

YORK UNIVERSITY, TORONTO 